machines. Laurence Devillers a ainsi préconisé de limiter les développements en matière de robots et d'algorithmes à ce qui serait le plus utile, pour les personnes âgées, les personnes moins âgées, pour nous faciliter la vie, mais sans nous rendre trop paresseux, ni trop dépendants des machines ${ }^{17}$.

En conclusion sur ce thème de l'interaction homme-machine, il a été regretté que les pouvoirs publics soient parfois mal informés. En particulier, selon Laurence Devillers, il faut réagir très clairement contre les implants cérébraux, s'ils ne sont pas justifiés par l'intention de réparer ou de traiter une maladie. Et il est inquiétant de voir que certaines institutions puissent être sensibles à des discours prônant l'eugénisme ou l'augmentation de l'homme, sous prétexte de baisse du QI moyen ou de compétition avec l'intelligence artificielle.

\title{
Intelligence artificielle et Insertion dans les réseaux sociaux
}

C'était un sujet trop vaste pour être traité dans le temps disponible. Et son rapport avec le cœur du thème central du colloque, voire avec celui de l'intelligence artificielle, n'est pas immédiat. Les interventions et la discussion ont cependant pu aborder plusieurs points.

Antoine Bordes rappelle l'un des caractères des réseaux sociaux : une plateforme telle que Facebook permet à des milliards d'entités d'échanger des informations de façon quasiment instantanée ; ces entités sont des personnes, mais aussi des groupes politiques, des journaux, des entreprises, voire des algorithmes ...; l'échelle de cet énorme espace d'expression, de liberté, qui, de plus, s'auto-développe, engendre des phénomènes qu'on ne prévoyait pas. L'observation et éventuellement, si nécessaire, le contrôle de ce qui se passe dans cet espace est difficile, compte tenu de sa taille mais aussi de la rapidité de son fonctionnement. La détection de contenus inappropriés (fausses nouvelles, faux comptes, incitations à la haine et autres propos interdits par la loi, ...) a d'abord été fondée sur des signalements : le contenu suspect est signalé à une équipe de vérifieurs qui décident s'il est ou non conforme aux règles de la plateforme, règles qui sont publiques et qui peuvent donc être confrontées aux différentes législations. En cas de non-conformité, ces vérifieurs décident éventuellement du retrait du contenu incriminé, mais bien sûr si ce contenu a été signalé c'est qu'il a déjà été vu et donc vu, a priori, par un grand nombre de personnes ... L'intelligence artificielle est apparue comme un outil pouvant aider ${ }^{18}$ un tel processus, notamment pour le rendre plus rapide. Mais d'un autre côté, de telles interventions se heurtent à un principe, qui est que l'on n'est pas là pour faire de la censure. La solution serait peut-être de rechercher comment faire comprendre au plus vite aux récepteurs que

\footnotetext{
${ }^{17}$ A ce propos a été cité le rapport récent de la mission Villani, qui préconise un usage raisonné des NTIC, y compris pour des raisons écologiques, du fait de leur coût élevé en énergie.

${ }^{18} \mathrm{La}$ décision quant au retrait devant toutefois rester humaine.
} 
tel ou tel contenu n'est pas fiable sans le censurer. Il s'agirait donc, dans l'idéal, de trouver des moyens pour attacher à l'information elle-même, une certaine valeur de fiabilité.

Laurence Devillers reprend ce thème, en évoquant un certain scepticisme devant ce qu'elle qualifie de scotchs, qui seraient mis ainsi sur certains contenus. Car le nombre de fakenews observées sur les réseaux sociaux, d'informations qui ne sont pas avérées, lui parait littéralement atterrant. Le web est apparu au début comme un formidable outil de mise à la disposition du plus grand nombre des connaissances acquises par l'humanité. Mais très vite, constate-t-elle, une grande anarchie s'est faite jour, poussant en avant des contenus qu'il est très difficile d'évaluer : pour faire le tri entre ce qui est avéré, pas avéré, ou carrément n'importe quoi, il faut être très bien outillé. On pourrait craindre - une hypothèse certes extrême- que ce développement anarchique nous échappe, entraîne une prolifération de fausses connaissances, de fausses idées, de faux amis, de faux algorithmes, et rende le web inutilisable. Une telle issue pourrait être partiellement une conséquence d'une révolte collective plus ou moins consciente devant la tendance d'une société hyper-connectée à nous enfermer. Et Laurence Devillers de citer un des angles d'attaque de cette tendance, qui est celui de notre santé : avec un scénario où nous serions environnés par une multitude d'objets connectés qui nous aideraient théoriquement à bien vivre, mais qui en fait nous piègeraient en décidant pour nous. Pourra-t-on supporter cela? N'y-a-t-il pas un risque d'explosion?

Francis Eustache fait alors remarquer que ce développement des réseaux sociaux associé à l'utilisation parfois effrénée d'Internet constituent des changements majeurs, dont certaines générations ont pleinement conscience, car elles ont connu l'état antérieur. Mais pour les plus jeunes malheureusement, il n'en est pas ainsi. Un des points caractéristiques de ce développement est l'externalisation de la mémoire. La mémoire est de plus en plus externalisée. Bien sûr, ce n'est pas nouveau dans l'histoire de l'humanité. Mais l'ampleur du phénomène est sans précédent. Quelles en sont les conséquences ? Notamment sur le plan psychologique, l'exigence, la nécessité d'une mémorisation interne disparaissant peu à peu ? Francis Eustache avance la nécessité de réfléchir sérieusement à cette évolution de l'équilibre entre mémoire interne et mémoire externe. Concernant toujours les conséquences psychologiques, que dire aussi de la prégnance, dans les réseaux sociaux, d'une sollicitation permanente faisant perdre le contrôle de la situation, que dire de la prégnance d'un fonctionnement de type stimulus-réponse excluant tout temps de réflexion? Alors que pour nombre d'opérations, l'assimilation du contenu d'un ouvrage très pointu par exemple, nous avons besoin, pour pouvoir les accomplir avec efficacité, de temps et d'un certain isolement. Cette dernière réflexion amène Francis Eustache à s'interroger sur ce qu'il en est dans l'action militaire.

Gérard de Boisboissel répond qu'effectivement la possibilité technique pour les soldats de se connecter à tout moment à leurs proches, nouvelle dans l'histoire militaire, poserait problème si elle était autorisée ; les jeunes ont le réflexe d'utiliser constamment leur smartphone dans la vie civile, pour communiquer avec leurs familles et leurs amis, et ils 
ont parfois du mal à comprendre que cet usage puisse être contrôlé. Pourtant, les raisons de ce contrôle sont multiples et assez évidentes : tout d'abord pour la préservation du moral du combattant projeté en opération, pour l'émergence et le maintien d'un esprit de groupe et de sa cohésion, et enfin pour de simples raisons de sécurité, l'adversaire pouvant avoir les moyens de détection appropriés et d'intrusion dans les réseaux. Gérard de Boisboissel aborde alors un autre point important lié à la connectivité : il est clair que dès à présent et a fortiori dans l'avenir, tous les équipements militaires déployés et les personnels mobilisés dans une opération feront partie d'un système global interconnecté, et produiront, transmettront, exploiteront un volume considérable de données, images, vidéos, etc. C'est une tendance qui lui semble inexorable et qui implique que l'art de conduire les opérations militaires doit s'adapter. Il s'agit d'un changement majeur qui n'est pas sans poser un certain nombre de problèmes nouveaux. Le problème de la sécurisation de ces réseaux, même s'ils sont non-ouverts sur le monde extérieur; le problème de la saturation $d u$ spectre, lié au volume des données à transmettre, un soldat n'ayant pas une fibre optique derrière lui. Enfin, le problème de l'exploitation de toutes ces données : qu'est-ce qu'on en fait, qui y a accès? Telle image, prise par un robot, doit-elle remonter au chef de groupe, de section, au capitaine, etc... Or le principe d'horizontalité à la base des réseaux peut entrer en contradiction avec le principe de subsidiarité nécessaire à la sécurité et à l'efficacité de l'organisation et de la prise de décision militaires ${ }^{19}$ : certaines informations ne doivent être transmises, au moins dans un premier temps, qu'aux seuls échelons pour lesquelles elles seront utiles dans l'action et qui ont la responsabilité de la conduite de l'opération. Aux échelons désignés de traiter et, pour ne pas encombrer les échelons supérieurs, il faudra activer un filtre des données qui sera fonction de la phase en cours de l'action militaire et de l'importance des données. L'IA pourra aider à la gestion dynamique de ce filtre. Et Gérard de Boisboissel de conclure : La bonne résolution de ce problème est un vrai enjeu de l'interconnexion des systèmes futurs.

\section{Conclusion}

Raja Chatila conclut le débat en résumant ce qu'il retient des interventions sur les quatre grands thèmes :

- La possibilité d'une conscience artificielle : non, cela n'existe pas, voire cela ne peut pas exister, aujourd'hui c'est un concept qui n'aurait pas de sens pour une machine.

- L'éthique et les décisions prises par les machines : la responsabilité sera toujours celle des humains qui ont conçu, déclenché et contrôlé la machine.

- Les interactions et les interdépendances hommes-machines : sur l'intégration de l'humain et de la machine par le biais de l'augmentation, il relève une alerte sur cette intégration qui transforme l'homme et présente des dangers, même si elle peut aussi être bénéfique.

${ }^{19}$ Ce problème se pose bien sûr également dans d'autres contextes. 
- L'insertion des machines dans les réseaux sociaux : elle pose plusieurs problèmes, notamment : l'externalisation de notre mémoire, qui transforme nos mécanismes de pensée; la difficulté de contrôler les réseaux, en évitant à la fois censure et manipulation ; la connexion vers l'extérieur, qui nous extirpe de notre réalité; et la diffusion inopportune des données collectées. 